\title{
A rare type of right atrial tumor in a 66-year-old woman
}

\author{
Alexander Lauten, MD, Justus T. Strauch, MD, Jens Wippermann, MD, and Thorsten Wahlers, MD, Cologne, Germany
}

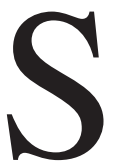

olid cardiac neoplasms are rare; however, depending on their location, they can be associated with serious complications. Fibroelastomas are endocardium-derived tumors that can develop in any chamber of the heart. A valvular position is known, with an appearance most often on the aortic valve and equal distribution on semilunar and atrioventricular valves. Located at the right side of the heart, the tumor generally remains asymptomatic. When sufficient in size, it might interfere with valvular function and hemodynamics and lead to secondary pulmonary embolization.

\section{Clinical Summary}

A 66-year-old woman was referred to us by the cardiology department with a recently diagnosed right atrial mass. The tumor had been revealed during a screening echocardiography before an elective orthopedic operation. A magnetic resonance imaging study showed late enhancement of the structure measuring $2.8 \times 2.5 \mathrm{~cm}$ with a broad base at the right atrial free wall and, although rarely located in the right heart, highly suspicious of an atrial myxoma (Figure 1). In the presence of chronic atrial fibrillation, thrombus formation was considered as a differential diagnosis. Over a period of 3 months, a marked progression in size was noted in repeated studies, and the patient was referred to us for urgent operative removal of the tumor. On admission, physical examination of the cardiopulmonary system was normal, apart from arterial hypertension. A dual-chamber pacemaker had been inserted 7 years earlier for atrioventricular block.

Removal of the tumor was performed through a standard right atriotomy after bicaval cannulation for cardiopulmonary bypass. Intraoperatively, the tumor presented as a gelatinous and fibrous conglomerate attached by a small base to the anterior atrial free wall just above the tricuspid annulus, causing first-degree insufficiency of the valve (Figure 2). Removal of the tumor, including the basic connection to the heart, was uneventful, and after closure of the atriotomy, the patient was easily weaned from cardiopulmonary bypass. After an uncomplicated postoperative course, she was discharged home 7 days after the operation on an oral anticoagulation regimen. Histologic evaluation of the intraoperative specimen revealed a papillary fibroelastoma of the right atrial endocardium.

From the Department of Cardiothoracic Surgery, University Hospital Cologne, Cologne, Germany.

Received for publication Aug 25, 2006; accepted for publication Aug 31, 2006.

Address for reprints: Alexander Lauten, MD, Department of Cardiothoracic Surgery, University Hospital of Cologne, Kerpener Str. 62, 50924 Cologne, Germany (E-mail: alexander.lauten@uk-koeln.de).

J Thorac Cardiovasc Surg 2007;133:251-2

$0022-5223 / \$ 32.00$

Copyright (C) 2007 by The American Association for Thoracic Surgery doi:10.1016/j.jtcvs.2006.08.069

\section{Discussion}

Solid masses of the right atrium are rarely found incidentally by using routine echocardiography at an early stage before causing clinical symptoms. Because diagnostic investigations are usually limited to imaging studies, a precise primary diagnosis is difficult to $\mathrm{m}$ ake. The presence of a right atrial mass is highly suspicious of a thrombus, either passing from a deep venous compartment to the pulmonary circulation or caused by pacemaker leads. ${ }^{1}$ Differentiation between thrombus formation and tumors arising from cardiac structures is possible on the basis of morphologic criteria, such as verification of a stalk base, or on the basis of proof-of-enhancement phenomena in magnetic resonance imaging studies. These criteria, however, are unsafe, and final diagnosis of the type and benign or malignant status of the tumor can only be made by means of histologic evaluation.

In consideration of a differential diagnosis, the atrial myxoma is the most likely of a variety of cardiac tumors. Although typically located in the left atrium, myxomas have also been found in the right atrium in a substantial number of patients. ${ }^{2}$ After myxoma and lipoma, papillary fibroelastomas represent the third most common type of benign cardiac tumors, accounting for less than $10 \%$ of primary cardiac tumors. ${ }^{3}$ Although these tumors can arise from any endocardial surface, they most often originate from the valvular endocardium, with almost equal frequencies on semilunar and atrioventricular valves. ${ }^{4}$

\section{Conclusions}

Papillary fibroelastomas of the right atrium are extremely rare; only 3 cases have been reported in the literature. Because the

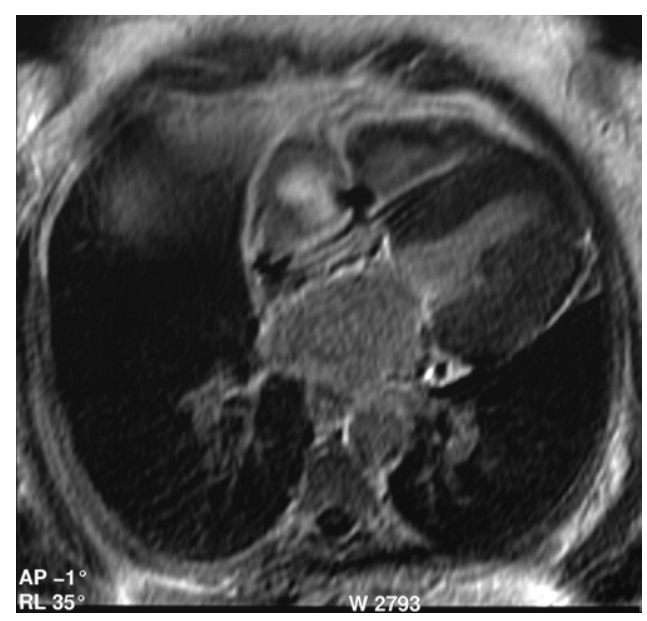

Figure 1. Preoperative magnetic resonance image showing an enhanced solid tumor originating from a broad base at the anterior right atrial wall. On the basis of morphologic criteria, atrial myxoma seems most likely. 

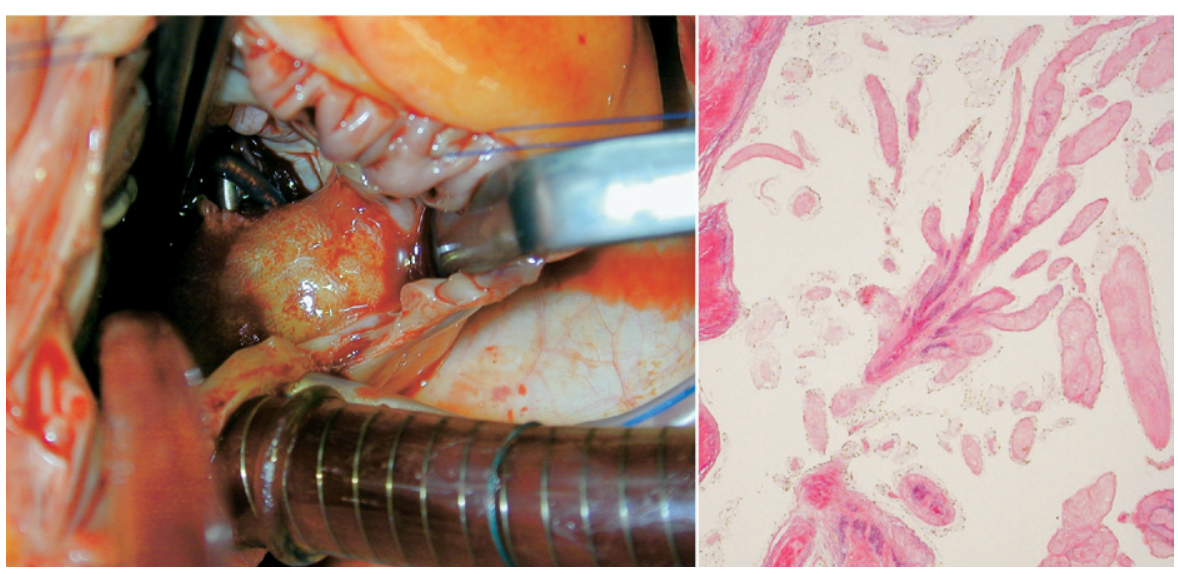

Figure 2. Intraoperative specimen and hematoxylin and eosin-stained histologic gross section. The tumor presents with numerous avascular papillary fronds covered by a single-layer endothelium.

tumor is benign, treatment requirements are based on its potential for obstructive effects and embolic events.

\section{References}

1. Chartier L, Bera J, Delomez M, Asseman P, Beregi JP, Bauchart JJ, et al. Free floating thormbi in the right atrial heart: diagnosis, management and prognostic indexes in 38 consecutive patients. Circulation. 1999; 99:2779-83.

2. Reynen K. Cardiac myxomas. N Engl J Med. 1995;333:1610-7.

3. Howard RA, Aldea GS, Shapira OM, Kasznica JM, Davidoff R. Papillary fibroelastoma: increasing recognition of a surgical disease. Ann Thorac Surg. 1999;68:1881-5.

4. Edwards FH, Hale D, Cohen A, Thompson L, Pezzella AT, Virmani R. Primary cardiac valve tumors. Ann Thorac Surg. 1991;52:1127-31.

\title{
Valve-sparing aortic root replacement in a patient with a rare connective tissue disorder: Arterial tortuosity syndrome
}

\author{
Tomaso Bottio, MD, PhD, Gianluigi Bisleri, MD, Paolo Piccoli, MD, and Claudio Muneretto, MD, Brescia, Italy
}

\section{Supplemental material is avail-} able online.

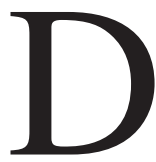

ilation of the aortic root and the ascending aorta is a well-known cardiovascular manifestation in pediatric patients with underlying connective tissue disorders, ${ }^{\text {E1 }}$ often leading to aortic valve incompetence and to aortic dissection risk. The Bentall procedure, a preventive operation, is a low-risk operation with considerably durable out-

From the Department of Cardiovascular Surgery, University of Brescia Medical School, Brescia, Italy.

Received for publication June 23, 2006; accepted for publication Aug 25, 2006.

Address for reprints: Tomaso Bottio, MD, Department of Cardiovascular Surgery, University of Brescia Medical School, Piazza Spedali Civili, 1, Brescia, Italy 25100 (E-mail: bottio@med.unibs.it).

J Thorac Cardiovasc Surg 2007;133:252-4

$0022-5223 / \$ 32.00$

Copyright $\odot 2007$ by The American Association for Thoracic Surgery doi:10.1016/j.jtcvs.2006.08.050 comes. ${ }^{1}$ Alternatively, other more conservative techniques have been proposed: aortic root remodeling and reimplantation., ${ }^{2,3}$

Arterial tortuosity syndrome (ATS) is a rare condition with an autosomal recessive inheritance characterized by tortuosity, elongation, stenosis, and aneurysm formation in the major arteries. ${ }^{4}$ Approximately 35 patients from 21 families have been reported thus far worldwide. This is the first case ever reported in the literature of a patient with ATS undergoing aortic root surgery with sparing of the aortic valve.

\section{Clinical Summary}

The patient, a 19-year-old man, was of a consanguineous family. One older sibling had ATS, as well as 2 of 3 cousins. ${ }^{4}$ At birth, he experienced a cardiac arrest. Dilation and elongation of the ascending aorta and arch was observed, followed by abrupt narrowing of the descending thoracic aorta; pulmonary arteries were aneurysmal and tortuous. A mutation in a third member of the SLC2A family (SLC2A10) was observed in this child. According to clinical features and genetic mapping, diagnosis of ATS was accomplished. ${ }^{4}$ In February 2004, the aortic annulus, the sinus portion of the ascending aorta, the sinotubular junction, and the ascending aorta measured $25,38,27$, and $30 \mathrm{~mm}$, respectively. At 